\title{
Range cell migration compensation in inverse synthetic aperture radar
}

Piotr Serafin, Czesław Leśnik, Kacper Zieliński

Piotr Serafin, Czesław Leśnik, Kacper Zieliński, "Range cell migration compensation in inverse synthetic aperture radar," Proc. SPIE 11442,

Radioelectronic Systems Conference 2019, 114421F (11 February 2020); doi: $10.1117 / 12.2565753$

SPIE. Event: Radioelectronic Systems Conference 2019, 2019, Jachranka, Poland 


\title{
Range cell migration compensation in inverse synthetic aperture radar
}

\author{
Piotr Serafin *a , Czesław Leśnik ${ }^{\mathrm{a}}$, Kacper Zieliński ${ }^{\mathrm{a}}$ \\ ${ }^{a}$ Military University of Technology, Faculty of Electronics, Institute of Radioelectronics, \\ 00-908 Warszawa, ul. gen. Sylwestra Kaliskiego 2, Poland
}

\begin{abstract}
Inverse synthetic aperture radar is a system allowing for acquiring of high resolution images of moving objects. The image synthesis algorithms base on coherent integration of observed object's echo signals, which requires the exact knowledge of the object's movement parameters in order to compensate two fundamental phenomena: echo's initial phase modulation and range cell migration of the signal. The range cell migration reduces maximal achievable resolution of the radar image and requires compensation based on the knowledge of the object's trajectory. Application on the second order keystone transform allows to perform the compensation without the knowledge of the parameters, however the shift of the location of the minimum range to the object. In this paper results of research on range migration compensation method applying the second order keystone transform are presented.
\end{abstract}

Keywords: inverse synthetic aperture radar, ISAR, range migration, range cell migration compensation, keystone transform

\section{INTRODUCTION}

Aperture synthesis technique [1] allows to create high resolution radar images in azimuth domain. It bases on exploiting the changes of the echo signals Doppler phase present due to the mutual movement between the object being imaged and the radar sensor observing it. The exact knowledge of the range changes function, the range curve, allows to perform the coherent integration of received signals and create the radar image.

In classic synthetic aperture radar (SAR) systems the imaged objects are assumed to be stationary and located on the surface of the ground, while the sensor, usually mounted on board of an aircraft or a spacecraft, moves along a set track. In this situation there is a possibility of determining the sensor movement's parameters with relatively high precision allowing to recreate the form of the range curve, and the phase correction function called the SAR reference function.

In inverse SAR (ISAR) systems, the sensor is usually immobile and the object moves in the radar's observed space. In Fig. 1. an exemplary geometry of the ISAR system is presented. The principle of such system is similar to the principle of SAR in its classic configuration. The radar sends sounding signals $s_{T}(t)$ with a central frequency $f_{0}$, which reach the observed object. The echo signals $s_{R}(t)$, return to the radar after a delay time $t_{o p}$ resulting from the distance to the object $R$

$$
t_{o p}=\frac{2 R}{c},
$$

where $c$ is the electromagnetic propagation speed.

Therefore, it can be assumed that the echo signal from a single point-like object is a delayed and scaled in amplitude copy of the sounding signal. If the analytic form of the sounding signal can be described as

$$
s_{T}(t)=A(t) \exp \left(j 2 \pi f_{0} t\right),
$$

where $A(t)$ is the function describing the complex amplitude of the sounding signal in the base band, $f_{0}$ is the central (carrier) frequency of this signal, then the received signal will take the following form

*piotr.serafin@wat.edu.pl; phone +48 26183 7593; fax +48 261837461

Radioelectronic Systems Conference 2019, edited by Piotr Kaniewski, Jan Matuszewski, Proc. of SPIE

Vol. 11442, 114421F · C 2020 SPIE · CCC code: 0277-786X/20/\$21 · doi: 10.1117/12.2565753 


$$
s_{R}(t)=s_{T}\left(t-t_{o p}\right)=A\left(t-t_{o p}\right) \exp \left[j 2 \pi f_{0}\left(t-t_{o p}\right)\right] .
$$

After converting this signal to the base band, it takes the form

$$
s_{R}(t)=A\left(t-t_{o p}\right) \exp \left(-j 2 \pi f_{0} t_{o p}\right) .
$$

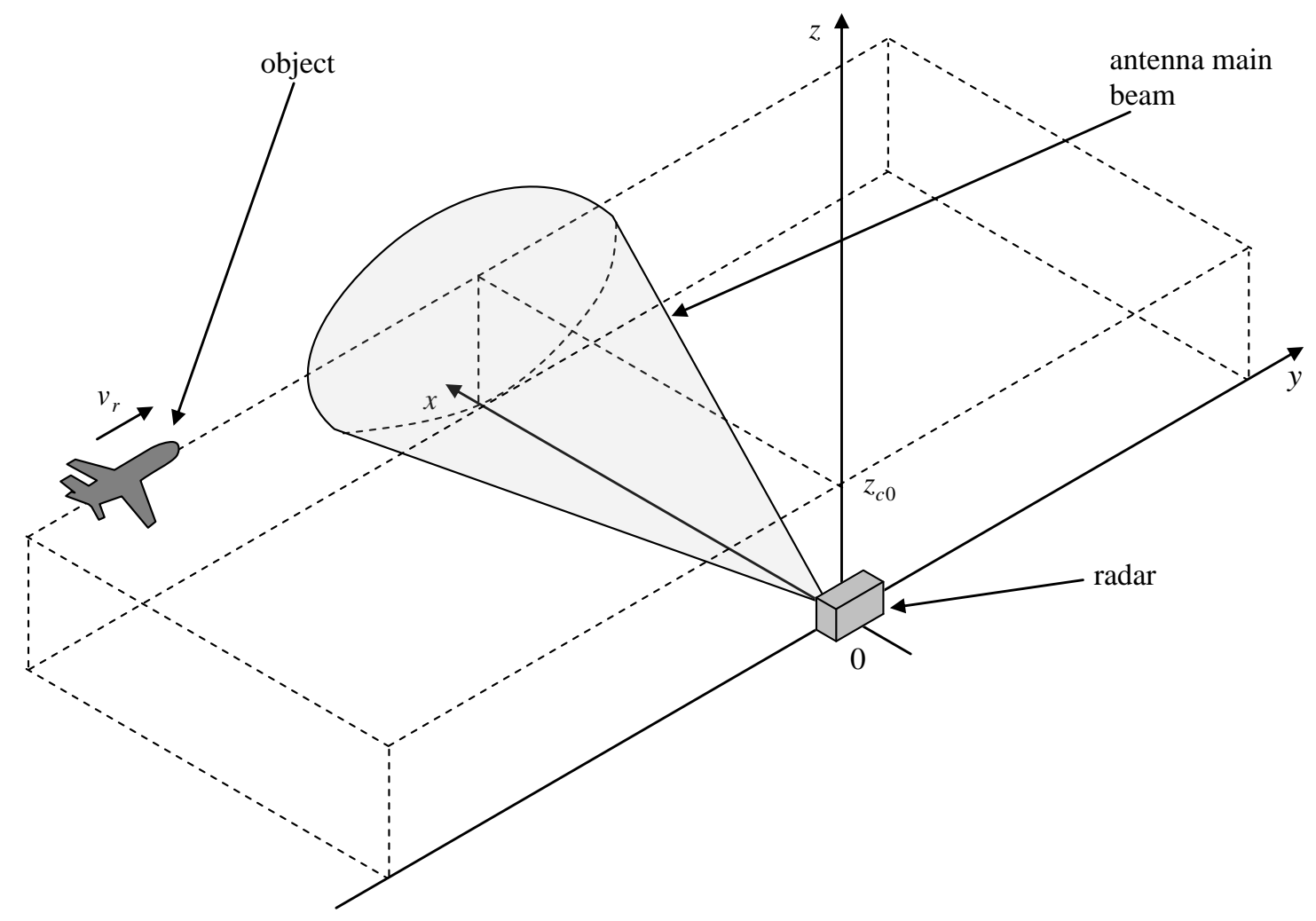

Figure 1. ISAR system geometry

\section{RANGE CELL MIGRATION}

The radar sends consecutive sounding pulses and receives echo signals in consecutive discrete moments of time $\tau$, therefore in radar signal processing algorithms two time domains [1] are defined: so called "fast time" $t$ associated with the propagation of the electromagnetic waves from the radar to the object and back to the radar, and the "slow time" $\tau$ associated with the movement of the object.

If it can be assumed that in the slow time the radar sends the same sounding signals, then the two-dimensional received signal may be written in the following form

$$
s_{R}(t, \tau)=A\left[t-t_{o p}(\tau)\right] \exp \left[-j 2 \pi f_{0} t_{o p}(\tau)\right]=A\left[t-\frac{2 R(\tau)}{c}\right] \exp \left[-j 2 \pi f_{0} \frac{2 R(\tau)}{c}\right],
$$

where $R(\tau)$ is the range curve function, whose shape depends on the system geometry and the velocity of the observed object. In a general case this function which is a distance from the radar to the observed object may be described by the following formula 


$$
R(\tau)=\sqrt{\left[x_{R}-x_{C}(\tau)\right]^{2}+\left[y_{R}-y_{C}(\tau)\right]^{2}+\left[z_{R}-z_{C}(\tau)\right]^{2}},
$$

where $\left[x_{R}, y_{R}, z_{R}\right]$ are the radar's coordinates and $\left[x_{C}(\tau), y_{C}(\tau), z_{C}(\tau)\right]$ are the slow time-dependent coordinates of the moving object.

If $\left[v_{C x}, v_{C y}, v_{C z}\right]$ is the object's velocity vector and $\left[x_{C 0}, y_{C 0}, z_{C 0}\right]$ is its initial location, then the equation (6) can be written as follows

$$
R(\tau)=\sqrt{\left[x_{R}-x_{C 0}+v_{C x} \tau\right]^{2}+\left[y_{R}-y_{C_{0}}-v_{C y} \tau\right]^{2}+\left[z_{R}-z_{C 0}+v_{C z} \tau\right]^{2}} .
$$

If the coordinate system is consistent with Fig. 1, i.e. such where the radar is located at the origin $[0,0,0]$, and the object's velocity vector has the form of $v_{C}=\left[0, v_{C y}, 0\right]$, is assumed, then equation (7) can be simplified to the following form

$$
R(\tau)=\sqrt{x_{C_{0}}^{2}+\left(y_{C_{0}}+v_{C y} \tau\right)^{2}+z_{C 0}^{2}} .
$$

The received signals are sampled in both time domains: fast and slow. The slow time sampling is performed in a natural way by sending the consecutive sounding signals by the radar, and the sampling in the fast time is performed in an analog-to-digital converter in the radar receiver.

The sampled received signal takes the following form

$$
s_{R}(m, n)=A\left[m-m_{o p}\right] \exp \left[-j 4 \pi f_{0} \frac{\sqrt{x_{C 0}^{2}+\left(y_{C 0}+v_{C y} n T_{p}\right)^{2}+z_{C 0}^{2}}}{c}\right],
$$

where $T_{p}$ is the sounding signal repetition interval, $m$ is the fast time sample (range cell) number, $n$ is the slow time sample number (sounding period number).

The received by the radar echo signals are stored in the systems memory and processed as if they were received by a very long real aperture antenna of the length equal to the distance covered by the object during one coherent processing interval (CPI). The processing consists of coherent summing of the signals after their Doppler phase shift compensation. The ISAR image synthesis algorithm is described by the following formula

$$
S(k, l)=\sum_{b=\lfloor-B / 2\rfloor}^{\lfloor B / 2\rfloor} s_{R}(w, l+b) \exp \left[j 4 \pi f_{0} \frac{\sqrt{(w d R)^{2}+\left(v_{C y} b T_{p}\right)^{2}}}{c}\right],
$$

where $k$ is the number of the image point (pixel) in the $x$ direction, $l$ is the number of the image pixel in the $y$ direction, $B$ is the length of the synthetic aperture expressed in number of soundings, $w$ is the range cell number, that is the source of the raw signal for the current synthetic aperture element, $d R$ is the size of the range cell.

The phase compensation of the signals along the synthetic aperture is performed by the exponential in (10) and allows to maximize the sum of the echo signals that come from the point corresponding to the $(k, l)$-th pixel of the ISAR image.

Equation (9) shows that the change of the distance between the sensor and the object causes not only the change of the phase of the echo signal, but also changes its location in the fast time. This phenomenon is called the range cell migration [1-4] and influences the maximum achievable synthetic aperture length.

In Fig. 2 an exemplary 2-D magnitude graph of the echo signal from a single point-like object is presented. The change of location of the maximum of the signal in the fast time as the object moves in the slow time is visible. 


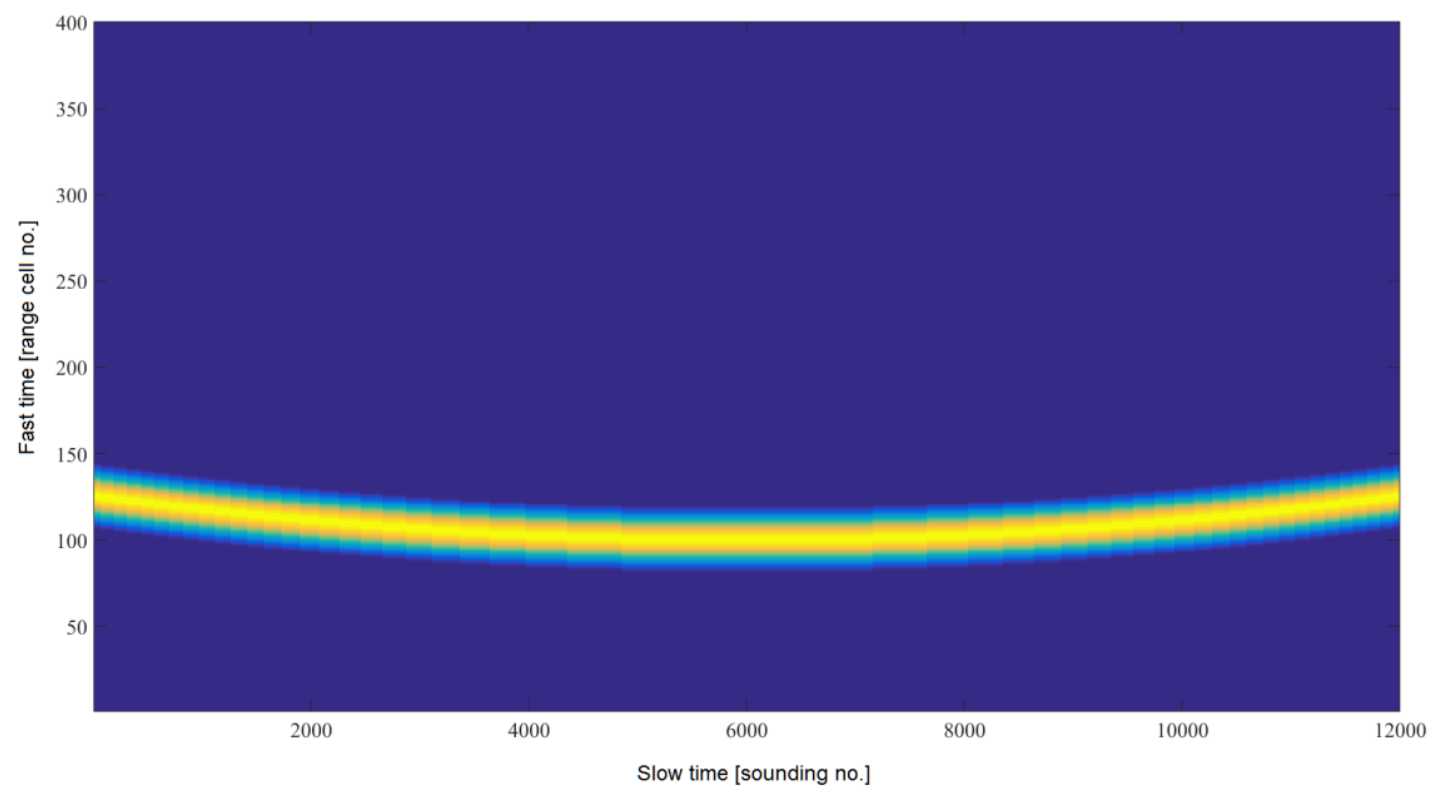

Figure 2. Raw ISAR signal

There are numerous methods of compensation for the echo signal range migration [1] in the SAR systems, but their application may be dependent on the image synthesis algorithm used in the system.

In the case of the algorithm described with eq. (10), the correction is performed by determining the number of the range cell $w$ from which the signal to be integrated is collected, which requires the knowledge of the geometry radar-object.

\section{THE KEYSTONE TRANSFORMATION}

Another method of range migration compensation in radar signal is the keystone transformation [2-4] that involves scaling the signal in the fast time spectrum domain. The canonical form of this transformation is used in classic radars to allow the coherent integration of echo signals from objects moving at high speeds [2, 3].

In order to compensate for the range migration the spectrum along the fast time $S_{R}(p, n)$ is determined where $p$ is the number of spectrum sample. In the next step for each frequency $f$ the scaling operation by $f_{0} /\left(f_{0}+f\right)$ is performed, which means that the new spectrum takes the form

$$
S_{R K}(p, n)=S_{R}\left(p, n \frac{f_{0}}{f_{0}+f}\right) .
$$

Thanks to this the change of the echo signals phase from pulse to pulse causes a proportional change of the location of those signals in the fast time. This correction does not require the knowledge of the velocity of the object and allows for the analysis of many objects simultaneously.

Due to the fact that in SAR systems the echo signals migrate accordingly to the range curve, this transformation does not yield to satisfactory results in image synthesis procedure.

In $[5,6]$ a method to apply the modified keystone transformation to the SAR system is presented. In this solution the second order keystone transformation was used.

The modification changes the scaling function, which takes the form of $\left[f_{0} /\left(f_{0}+f\right)\right]^{1 / 2}$. This allows to compensate for the migration despite the hyperbolic character of the range curve. However it must be noticed that the above scaling 
function form is correct only for the case when the object passes the radar at the middle of the observation time, i.e. when the range curve is symmetric with reference to the middle of this time interval.

In order to enable the correct compensation, the shift of the minimum of the range curve has to be taken into account. Assuming that the radar observes the object in a narrow azimuth sector it is possible to find this shift and apply it to the transformation, which takes the form of

$$
S_{R K 2}(p, n)=S_{R}\left[p, n\left(\frac{f_{0}}{f_{0}+f}\right)^{1 / 2}-n_{0}\right],
$$

where $n_{0}$ is the value of the range curve's minimum shift expressed in a number of soundings determined in the following way

$$
n_{0}=\frac{R_{0} \sin \theta_{0}}{v_{C y} F_{p}},
$$

where $R_{0}$ is the distance to the middle point of the object observation area, $\theta_{0}$ is the object middle observation angle, $F_{p}$ is the sounding pulse repetition frequency.

\section{SIMULATION RESULTS}

In order to verify the correctness of the presented assumptions simulation experiments have been carried out. In the experiments ISAR raw signals representing single point-like echo of an object moving with a specific velocity along a straight line trajectory have been generated.

In the simulation the following parameters were assumed: $f_{0}=3 \mathrm{GHz}, F_{p}=3 \mathrm{kHz}, R_{0}=1100 \mathrm{~m}, v_{C}=[0,40,0] \mathrm{m} / \mathrm{s}$, $\theta_{0}=3^{\circ}$ and $\theta_{0}=5^{\circ}$. In Fig. 3-6 the raw signal before and after the range migration compensation are presented. The observed point-like object have been moving pararelly to the $O Y$ of the assumed coordinate system.

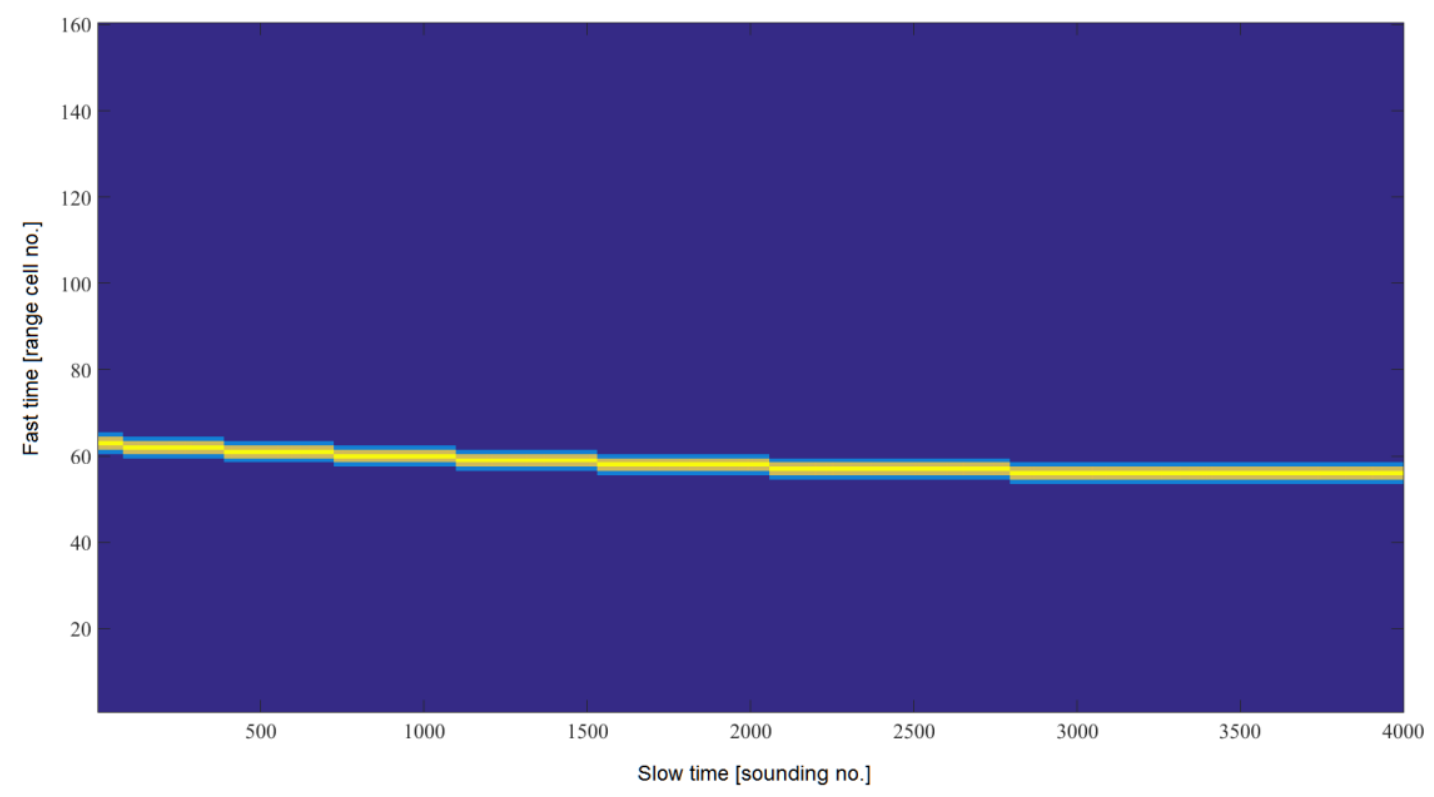

Figure 3. Raw ISAR echo signal before range migration compensation for $v_{C}=[0,40,0] \mathrm{m} / \mathrm{s}$ and $\theta_{0}=3^{\circ}$ 


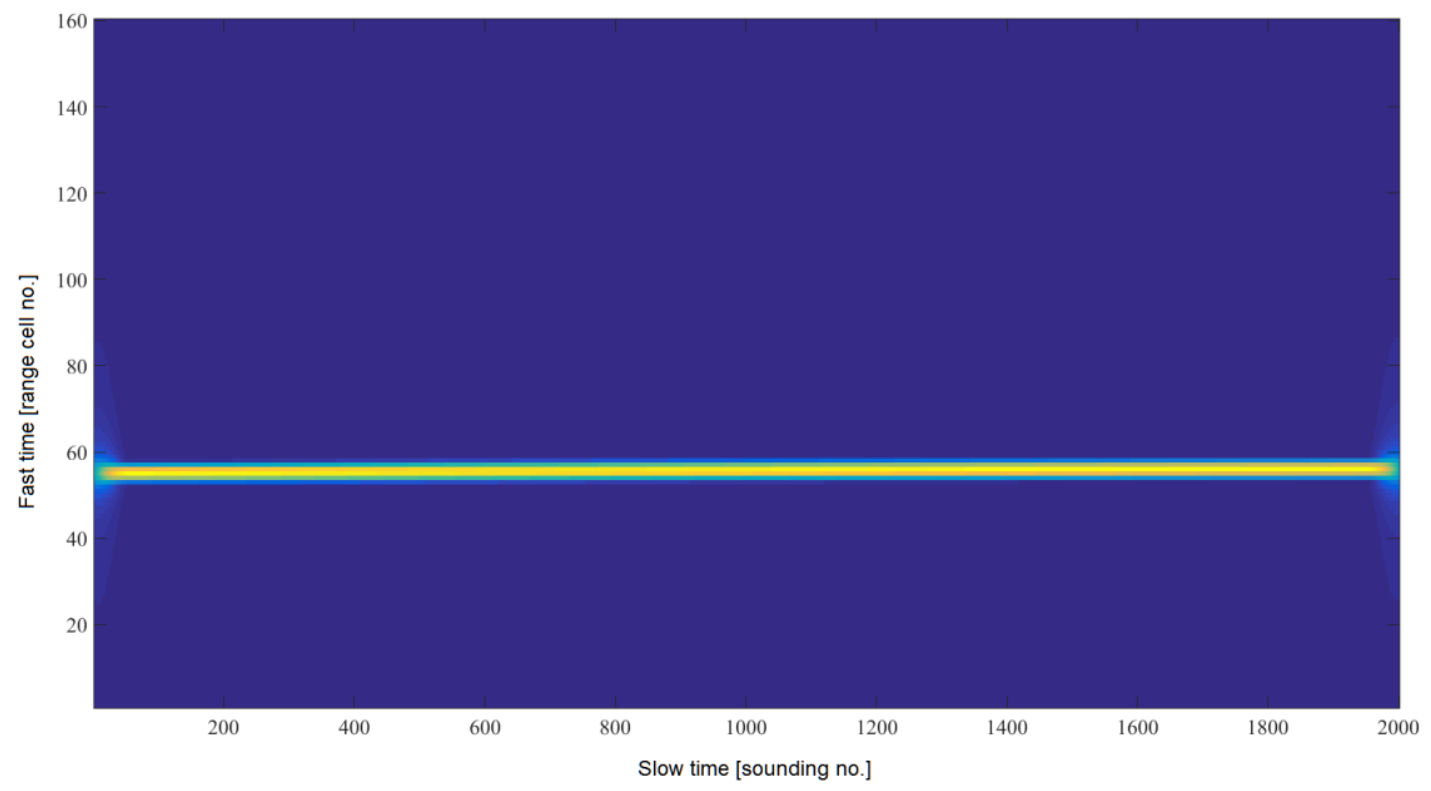

Figure 4. Raw ISAR echo signal after range migration compensation for $v_{C}=[0,40,0] \mathrm{m} / \mathrm{s}$ and $\theta_{0}=3^{\circ}$

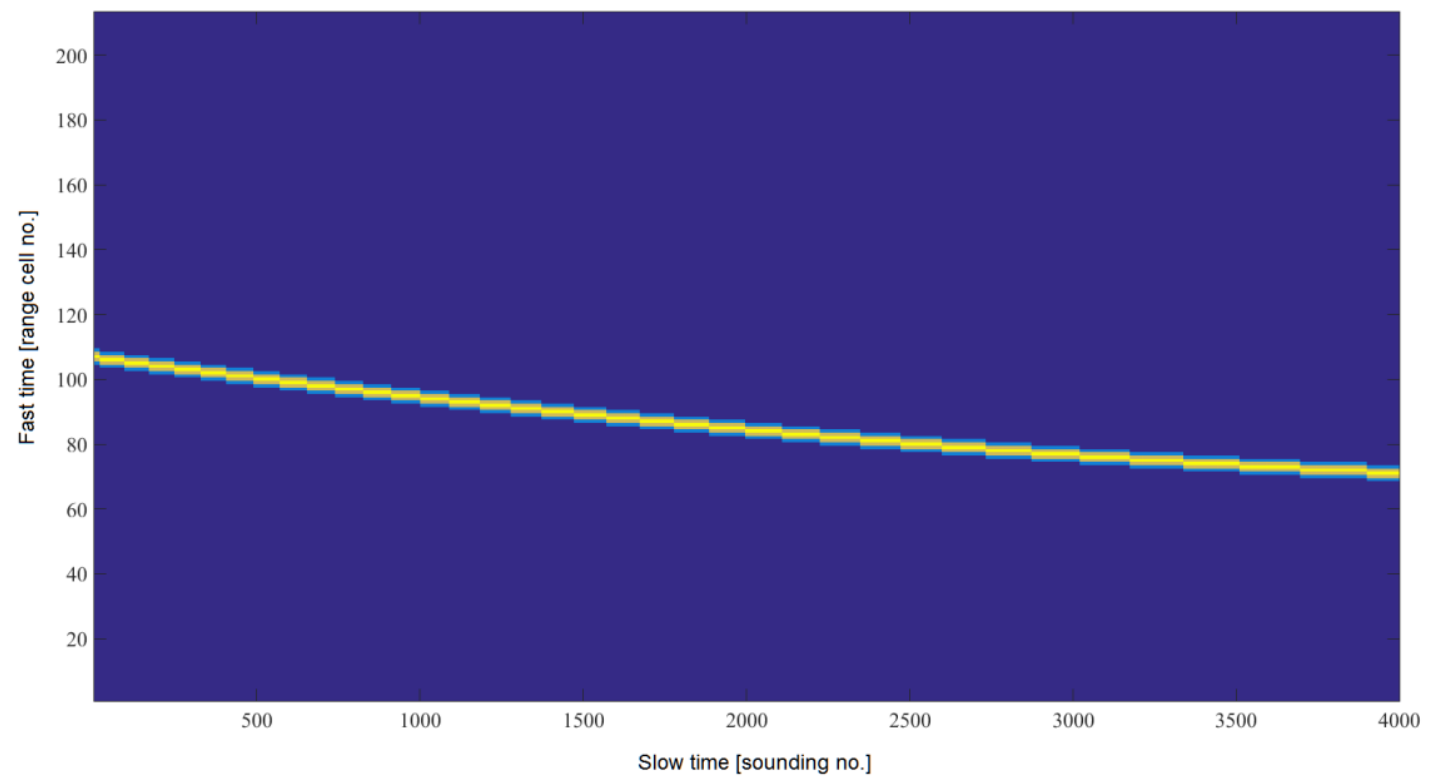

Figure 5. Raw ISAR echo signal before range migration compensation for $v_{C}=[0,40,0] \mathrm{m} / \mathrm{s}$ and $\theta_{0}=5^{\circ}$ 


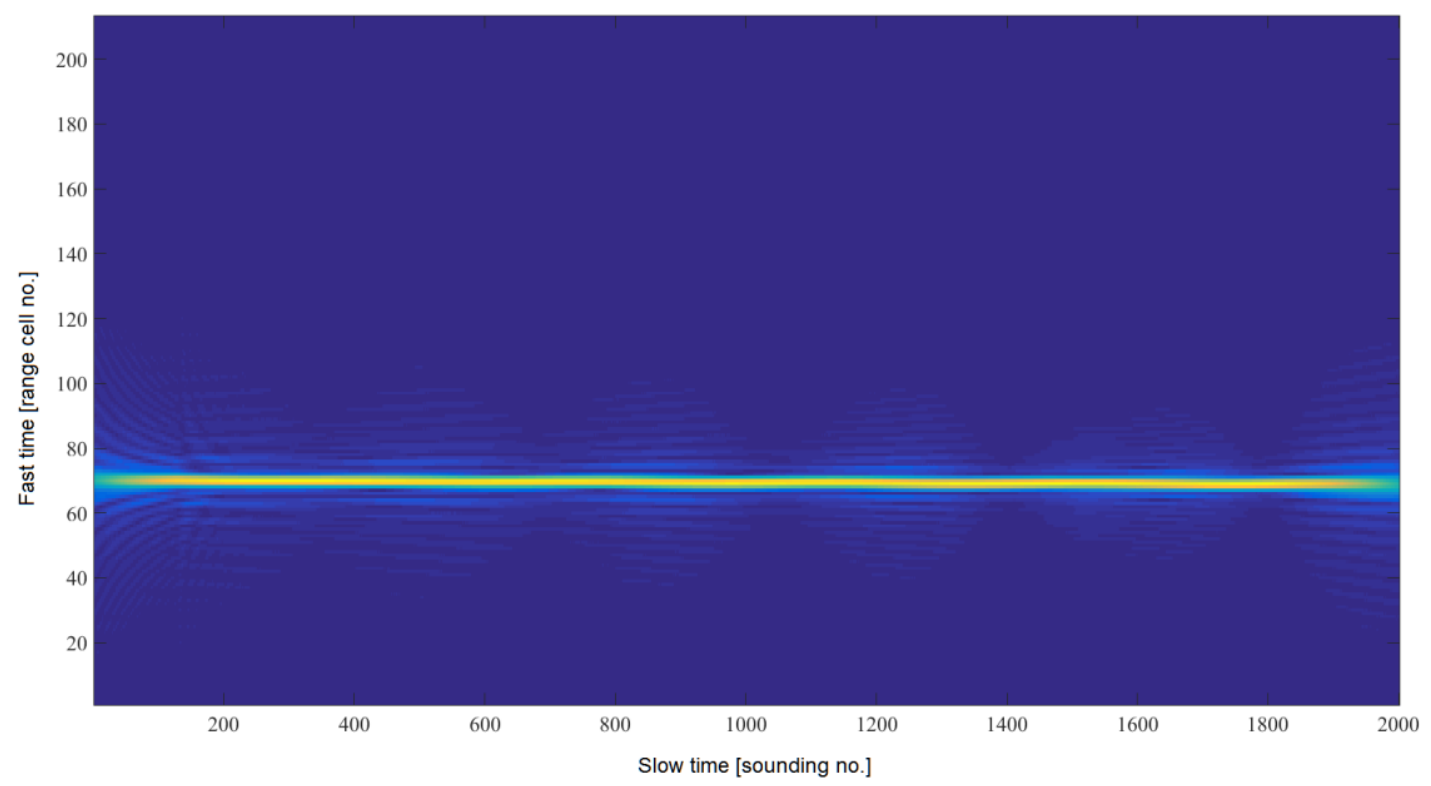

Figure 6. Raw ISAR echo signal after range migration compensation for $v_{C}=[0,40,0] \mathrm{m} / \mathrm{s}$ and $\theta_{0}=5^{\circ}$

In the graphs presented in Fig. 3 and 5 a significant range migration can be observed, which may render the image synthesis at its best achievable resolution difficult. After the compensation procedure (Fig. 4 and Fig. 6) the echo signal maximum is aligned along the slow time in one range cell, which allows to directly integrate the signals in subsequent sounding periods.

\section{SUMMARY}

In the paper the results of the simulation tests on range cell migration compensation method in an inverse synthetic aperture radar using the second order keystone transformation is presented. The transformation thanks to the spectrum scaling allows for elimination of the migration of the signal, however in the case of synthetic aperture systems an additional correction of the range curve minimum shift is needed.

\section{ACKNOWLEDGEMENTS}

This work has been financed by Military University of Technology, Warsaw, Poland in 2019 within the project PBS 659.

\section{REFERENCES}

[1] Wang B. C., [Digital Signal Processing Techniques and Applications in Radar Image Processing], Wiley, (2008).

[2] Perry R. P., DiPietro, R. C., Fante, R., P., "Coherent Integration with Range Migration Using Keystone Formatting", Proceedings of the 2007 IEEE Radar Conference, Boston, MA, April, 17-20. 863-868 (2007).

[3] Richards, M. A., "The Keystone Transformation for Correcting Range Migration in Range-Doppler Processing”, March, 28, 2014 https://radarsp.weebly.com/uploads/2/1/4/7/ 21471216/keystone.pdf (2014). 
[4] Yang L., Tao Z., Teng L. Zheng W., "Range migration compensation and Doppler ambiguity resolution by keystone transform", Proc. 2006 International Conf. on Radar, pp. 1-4, Oct. 2006.

[5] Kirkland, D.,: "Imaging moving targets using the second-order keystone transform", IET Radar, Sonar \& Navigation, vol. 5, Issue: 8, October 2011, 902-910, (2011).

[6] F. Zhou, R. Wu, M. Xing, and Z. Bao, "Approach for single channel SAR ground moving target imaging and motion parameter estimation" IET Radar Sonar Navigation., vol. 1, no. 1, pp. 59-66, February 2007. 